\title{
TRAUMA TORÁCICO: FATORES DE RISCO DE COMPLICAÇÕES PLEUROPULMONARES PÓS-DRENAGEM PLEURAL FECHADA
}

\author{
THORACIC TRAUMA: A RISK ANALYSIS OF PULMONARY COMPLICATIONS \\ FOLLOWING CLOSED TUBE THORACOSTOMY
}

\author{
Mauro José Pantoja Fontelles ${ }^{1}$ \\ Mario Mantovani, TCBC-SP ${ }^{2}$
}

\begin{abstract}
RESUMO: O objetivo do presente estudo foi avaliar a importância dos fatores de risco na gênese das complicações pleuropulmonares, pós-drenagem pleural fechada. Analisou-se, prospectivamente, no período de janeiro de 1998 a junho de 1999, um total de 167 pacientes submetidos à drenagem pleural fechada, sendo estratificados em dois grupos selecionados para um estudo de acompanhamento de coortes. Ao grupo controle, de 104 pacientes, não foi administrada antibioticoterapia e, no grupo experimental, de 63 pacientes, a cefalotina foi a droga utilizada. A idade no grupo-controle variou entre 13 e 53 anos $(26,8 \pm 8,9)$ e no grupo experimental entre 15 e 57 $(24,9 \pm 7,9)$, predominando o sexo masculino $(95,2 \%)$, nos dois grupos estudados. O trauma aberto incidiu em $92,8 \%$ dos pacientes, com predominância para as feridas por arma branca em $58,7 \%$, contra $24,6 \%$ de feridas por projétil de arma de fogo. As complicações pleuropulmonares estiveram presentes em 35 pacientes $(33,78 \%)$ do grupo controle, ao passo que, no grupo experimental, apenas 18 (28,6\%) evoluíram com este tipo de complicação. Não ocorreram óbitos em ambas as séries estudadas. Nas análises estatísticas, o modelo bivariado mostrou que o tipo de trauma e o tempo de drenagem pleural foram as variáveis que mais importância tiveram como fatores preditivos de complicações. Na análise de regressão logística multivariada, as variáveis tempo de internação, trauma fechado e volume de sangue drenado maior do que $500 \mathrm{ml}$, quando associadas, influenciaram de maneira positiva a ocorrência de complicações.
\end{abstract}

Descritores: Trauma; Trauma torácico; Drenagem pleural; Complicações pleuropulmonares.

\section{INTRODUÇÃO}

A terapêutica cirúrgica das lesões de órgãos intratorácicos tinha um campo bastante restrito até o início do século XX. Na época em que já se contabilizavam importantes avanços nos processos cirúrgicos para lesões abdominais, só alguns poucos procedimentos eram realizados para as afecções do tórax ${ }^{1}$. Por outro lado, o estudo do trauma torácico precede os princípios dos relatos históricos. Três casos semelhantes deste tipo de lesão foram descritos em papiro, ficando estes escritos atribuídos ao médico egípcio Imhotep, entre os anos 3.000 a 2.500 a.C. Este documento é conhecido como o relato científico mais antigo da história, estando as lesões ali citadas, descritas em uma ordem que posteriormente se tornaria tradicional: $a$ capite ad calcem, ou seja, da cabeça aos pés ${ }^{2}$.
Referências mais recentes sobre a epidemiologia do traumatismo torácico, mostram que, no ano de 1985, ocorreram 94 mil mortes acidentais nos Estados Unidos, eqüivalendo a 40 mortes por 100 mil habitantes e que, em outros 26 países, esta cifra sobe para 52 mortes, quando comparada a igual número da população ${ }^{3}$.

Atualmente, numa estatística alarmante, o trauma é a terceira maior causa de morte naquele país e a primeira quando considera-se a idade abaixo dos 40 anos. Ali, ocorrem cerca de 100 mil mortes causadas por acidente, a cada ano, com mais de 9 milhões de lesões incapacitantes. Aproximadamente $25 \%$ destas mortes são causadas por traumatismo fechado, correspondendo as lesões torácicas a 50\% de todas elas ${ }^{4}$.

Neste sentido, as feridas torácicas representam a causa de $20 \%$ de todas as mortes por trauma e mais do que $30 \%$

1. Mestre em Cirurgia do Trauma do Departamento de Cirurgia da Faculdade de Ciências Médicas da UNICAMP.

2. Professor titular e chefe da Disciplina de Cirurgia do Trauma do Departamento de Cirurgia da Faculdade de Ciências Médicas da UNICAMP.

Recebido em 16/11/1999

Aceito para publicação em 8/8/2000

Trabalho Realizado pela Disciplina de Cirurgia do Trauma do Departamento de Cirurgia da Faculdade de Ciências Médicas da UNICAMP - Campinas-SP. 
entre todos os tipos de lesões, mostrando que a mortalidade dos pacientes hospitalizados com apenas uma lesão isolada do tórax varia de $4 \%$ a $8 \%$, e aumenta para $10 \%$ a $25 \%$, quando um outro órgão é envolvido, elevando-se para $35 \%$, quando há comprometimento de múltiplos sistemas orgânicos 5,6 .

Embora a incidência de morte nas lesões torácicas ocupe lugar de destaque nas estatísticas mundiais, grande parte dos pacientes com este tipo de trauma pode ser tratada sem grandes procedimentos cirúrgicos, reservando-se a toracotomia para cerca de $10 \%$ a $20 \%$ do casos. Entretanto, aproximadamente um terço dos pacientes com lesões graves do tórax morre antes do atendimento hospitalar, e outros $20 \%$ apresentam morte tardia, em conseqüência das complicações pleuropulmonares de natureza infecciosa que, direta ou indiretamente, resultam deste tipo de violência $4,7,8$.

Isto posto, este estudo tem por objetivo, avaliar a importância dos fatores de risco envolvidos na gênese das complicações pleuropulmonares, nos pacientes acometidos por trauma isolado do tórax e submetidos à drenagem pleural fechada.

\section{MÉTODO}

Seguindo orientação do protocolo previamente estabelecido pela Disciplina de Cirurgia do Trauma do Departamento de Cirurgia da UNICAMP, foram admitidos, no presente estudo, 167 pacientes atendidos na Unidade de Emergência do Hospital de Pronto-Socorro Municipal de Belém, no período de janeiro/98 a junho/99, todos apresentando lesão isolada do tórax, com diagnóstico de hemotórax e/ou pneumotórax, e submetidos à drenagem pleural sob selo d'água. A técnica utilizada seguiu orientação como preconizada pelo Advanced Trauma Life Support $(\text { ATLS })^{9}$.

Duas coortes foram selecionadas para comparação. A primeira, definida como grupo controle (grupo I), foi constituída de 104 (62,3\%) pacientes, sem uso da antibioticoterapia. A segunda, grupo experimental, com 63 $(37,7 \%)$ pacientes, ao qual a cefalotina foi administrada na dosagem de 500mg, por via intravenosa, a cada seis horas, iniciada imediatamente antes da drenagem e continuada até 12 horas após a retirada do dreno ou, por no máximo, cinco dias.

Todas as informações foram arquivadas em banco de dados, utilizando-se o programa EPI INFO 6.04 (CDC Centers for Disease Control \& Prevention, USA), possibilitando o cruzamento e a comparação entre os grupos estudados. Em todas as análises estatísticas empregou-se o nível alfa de 5\% (p $£ 0,05)$. A correlação entre as variáveis, na análise bivariada, foi realizada através da aplicação do teste de qui-quadrado, em tabelas de contingência de dupla entrada, para os cruzamentos de interesse. Tratando-se com freqüências menores do que cinco ou com pequenas amostragens, o teste de qui-quadrado foi substituído pelo teste de probabilidade exata de Fisher. As complicações, atelectasia, pneumonia e empiema pleural foram consideradas como variáveis dependentes.

As variáveis correlacionadas com as constantes, representadas pelas complicações pleuropulmonares, foram: idade, tipo de trauma, agente vulnerante, hemotórax coagulado, tempo decorrido da lesão até o atendimento, presença de choque à admissão, tempo de drenagem e internação, Índices de trauma (Revised Trauma Score - RTS, Injury Severity Score - ISS, TRISS), presença ou não de atelectasias durante a evolução clínica.

A antibioticoterapia foi incluída no modelo estatístico com objetivo de verificar sua importância clínica, na presença dos fatores de risco, em evitar uma evolução desfavorável dos pacientes estudados.

A importância de cada variável, isoladamente, na determinação do prognóstico e sua associação com as demais variáveis estudadas, exigiram a utilização de técnicas de análise multivariada. A técnica de regressão logística foi utilizada para associar todas as variáveis de valor prognóstico e agrupá-las em um mesmo modelo. Utilizou-se o procedimento Stepwise ${ }^{10}$ para selecionar as variáveis que melhor explicaram a variável resposta "complicações pleuropulmonares". Os valores de "p" foram obtidos através do programa estatístico SAS (Statistical Analysis System).

Utilizou-se a odds ratio (razão de chance) para mensurar a associação entre um fator de risco e a ocorrência de complicações. Este procedimento consiste em uma expressão matemática que define a chance relativa de desenvolver uma complicação na presença de um determinado fator de risco, e toma por base a distribuição observada na tabela de contingência.

Neste trabalho, com objetivo de padronizar o diagnóstico das complicações pleuropulmonares, adotaramse os critérios propostos pelo North American Trauma Center. Foram consideradas: atelectasia lobular, lobar ou pulmonar; pneumonia ou broncopneumonia e empiema pleural $^{11-13}$.

A atelectasia foi diagnosticada pela interpretação dos achados radiográficos que tomou como parâmetro a identificação de imagens com base no broncograma aéreo, colapso segmentar ou lobar, sinal da silhueta e visualização das linhas do espaço pleural. A pneumonia foi identificada pelos sinais sistêmicos de infecção (febre, taquicardia, leucocitose); aparecimento ou piora radiológica do infiltrado pulmonar; expectoração de aspecto purulento. $\mathrm{O}$ empiema foi confirmado, tomando-se como base os achados clínicos de febre (temperatura axilar $>38^{\circ} \mathrm{C}$ ), sinais de toxemia do paciente, derrame pleural persistente com aspecto turvo e purulento do líquido coletado. Os achados radiológicos de líquido no espaço pleural e a identificação de microrganismos na secreção, caracterizaram o quadro ${ }^{14,15}$.

O derrame pleural, o pneumotórax e o hemotórax residuais foram considerados como complicações torácicas "menores", decorrentes de falha no sistema de drenagem $^{16}$.

O volume drenado de $50-100 \mathrm{ml} \mathrm{nas} 24$ horas, o aspecto seroso ou serossanguinolento do líquido coletado, ausên- 
cia de borbulhamento no frasco de drenagem por um período não inferior a 24 horas e controle clínico e radiológico, foram os critérios para retirada do dreno torácico. A presença de secreção purulenta foi indicativa da necessidade de cultura e prova de sensibilidade aos antimicrobianos.

Excluiu-se do presente estudo os pacientes com indicação de toracotomia, drenagem pleural realizada em outro hospital, lesões associadas localizadas em outras regiões anatômicas, história clínica de doenças preexistentes de manifestação aguda ou crônica, uso de antimicrobianos antes da ocorrência do trauma, idade abaixo de 13 anos, diagnóstico de gravidez e pacientes transferidos para outro hospital no curso do tratamento.

\section{RESULTADOS}

Todos os pacientes selecionados para o estudo tiveram indicada a drenagem pleural, durante a avaliação inicial, na unidade de emergência. Cento e cinqüenta e nove $(95,2 \%)$ pacientes eram do sexo masculino e oito $(4,8 \%)$ do sexo feminino. A faixa etária dos pacientes situou-se entre 13 e 57 anos. Cento e vinte $(71,9 \%)$ pacientes pertenciam à faixa etária mais jovem da população com idade compreendida entre 13 e 29 anos (Tabela 1).

Nos dois grupos estudados, $83(49,7 \%)$ pacientes apresentavam diagnóstico de hemotórax, em 16 (9,6\%) encontrou-se pneumotórax e $68(40,7 \%)$ eram portadores de hemopneumotórax (Tabela 2).
O intervalo de tempo decorrido do momento da agressão até o atendimento, com realização da drenagem pleural, variou de um período menor do que uma hora até mais do que 24 horas, com média \pm desvio-padrão de 3,6 $\pm 1,3$ horas. O registro da pressão arterial (PA) à admissão revelou que em $161(96,4 \%)$ pacientes os valores sistólicos estavam cima de $90 \mathrm{mmHg}$, inclusive, ao passo que, em seis $(3,6 \%)$, a pressão arterial sistólica era menor do que $90 \mathrm{mmHg}$.

Com base nos achados cirúrgicos, todos os doentes tiveram os índices de trauma calculados à admissão. O valor médio do RTS foi avaliado em 7,4 (5,4-7,6), o ISS com $10,1(09-16)$ e o TRISS $98,9 \%(97,0 \%$ - 99,0\%).

Tabela 1

Número de casos conforme a idade

\begin{tabular}{lcc|c}
\hline Idade (anos) & $\begin{array}{c}\text { Número } \\
\text { de casos }\end{array}$ & $\%$ & Acumulado \\
\hline $13-19$ & 46 & 27,5 & 27,5 \\
$20-29$ & 74 & 44,4 & 71,9 \\
$30-39$ & 30 & 17,9 & 89,8 \\
$40-49$ & 13 & 7,8 & 97,6 \\
$50-57$ & 04 & 2,4 & 100,0 \\
\hline Total & 167 & 100,0 & \\
\hline
\end{tabular}

Tabela 2

Número de casos conforme as indicações para toracotomia de acordo com o diagnóstico e o grupo estudado

\begin{tabular}{|c|c|c|c|c|}
\hline \multirow{2}{*}{ Indicação } & \multicolumn{2}{|c|}{ Sem Antibiótico $(n=104)$} & \multicolumn{2}{|c|}{ Com Antibiótico $(n=63)$} \\
\hline & № de casos & $\%$ & № de casos & $\%$ \\
\hline Hemotórax & 55 & 52,9 & 28 & 44,4 \\
\hline Arma branca & 30 & 28,9 & 14 & 22,4 \\
\hline Arma de fogo & 21 & 20,2 & 10 & 15,8 \\
\hline Contusão & 02 & 1,9 & 03 & 4,7 \\
\hline Outros & 02 & 1,9 & 01 & 1,5 \\
\hline Pneumotórax & 13 & 12,5 & 03 & 4,7 \\
\hline Arma branca & 08 & 7,7 & 02 & 3,2 \\
\hline Arma de fogo & - & - & - & - \\
\hline Contusão & 03 & 2,9 & - & - \\
\hline Outros & 02 & 1,9 & 01 & 1,5 \\
\hline Hemopneumotórax & 36 & 34,6 & 32 & 50,9 \\
\hline Arma branca & 26 & 25,0 & 18 & 28,8 \\
\hline Arma de fogo & 03 & 2,9 & 07 & 11,1 \\
\hline Contusão & 02 & 1,9 & 02 & 3,1 \\
\hline Outros & 05 & 4,8 & 05 & 7,9 \\
\hline
\end{tabular}


O volume de sangue coletado na cavidade pleural foi aferido para cada caso, variando de $0,0 \mathrm{ml}$ (volume desprezível) a $1.800 \mathrm{ml}$. A monitorização horária foi realizada para detectar persistência do sangramento e possível indicação de toracotomia.

O período de permanência do dreno variou de três a 17 dias, com média \pm desvio-padrão de 5,1 $\pm 2,8$ dias. Em $132(79,0 \%)$ pacientes, o tempo de drenagem variou entre três e cinco dias, ao passo que $35(21,0 \%)$ permaneceram com dreno além deste período de tempo. O tempo médio de drenagem no grupo de pacientes que não recebeu antibiótico foi de 4,8 82,6 dias, e no grupo de pacientes com antibioticoterapia o tempo médio foi de 5,6 $\pm 3,1$ dias.

A permanência da internação hospitalar variou de três a 18 dias, com a média \pm desvio padrão de $5,7 \pm 3,0$ dias. Observou-se que em $110(65,9 \%)$ pacientes, o tempo de internação variou de três a cinco dias e, em $57(34,1 \%)$, este período ficou compreendido entre seis e 18 dias de internação.

Quando avaliado o tempo de internação, verificou-se que, no grupo que não recebeu antibiótico a média de permanência hospitalar foi de $5,7 \pm 3,2$ dias e, no grupo com antibioticoterapia, este valor foi de 5,7 $\pm 2,9$ dias (Tabela 3 ).

Tabela 3

Número de casos conforme o tempo de internação e o grupo estudado

\begin{tabular}{l|c|c|c|c}
\hline $\begin{array}{l}\text { Tempo de } \\
\text { internação } \\
\text { (dias) }\end{array}$ & \multicolumn{2}{|c|}{$\begin{array}{c}\text { Sem Antibiótico } \\
(n=104)\end{array}$} & \multicolumn{2}{c}{$\begin{array}{c}\text { Com Antibiótico } \\
(n=63)\end{array}$} \\
\cline { 2 - 5 } & $\begin{array}{c}N^{o} \\
\text { de Casos }\end{array}$ & $\%$ & $\begin{array}{r}N^{o} \\
\text { de Casos }\end{array}$ & $\%$ \\
\hline 3 a 5 & 71 & 68,3 & 39 & 61,9 \\
6 a 10 & 26 & 25,0 & 19 & 30,2 \\
11 a 15 & 03 & 2,9 & 04 & 6,3 \\
16 a 18 & 04 & 3,8 & 01 & 1,6 \\
\hline Total & 104 & 100,0 & 63 & 100,0 \\
\hline
\end{tabular}

Do total de 167 pacientes estudados, $53(31,7 \%)$ cursaram com complicações pleuropulmonares. As atelectasias apresentaram maior predomínio, com incidência de 39 $(23,3 \%)$ pacientes, entre os quais apenas cinco $(2,9 \%)$ evoluíram com algum tipo de complicação infecciosa (pneumonia ou empiema). Os demais pacientes com atelectasia não apresentaram qualquer manifestação sistêmica sugestiva de infecção.

As pneumonias representaram a segunda maior incidência entre as complicações pleuropulmonares, com oito $(4,7 \%)$ pacientes, quando estudadas sem associação com outra complicação, ou $13(7,7 \%)$ pacientes, quando em associação com atelectasia ou empiema.
O empiema pleural incidiu em quatro $(2,3 \%)$ pacientes, quando avaliado de maneira isolada, ou em oito $(4,6 \%)$, quando associado com atelectasia ou pneumonia (Tabela 4).

Tabela 4

Número de casos conforme ds complicações pleuropulmonares

\begin{tabular}{lcc}
\hline Complicações Pleuropulmonares & No de Casos & $\%$ \\
\hline Atelectasia & 34 & 20,3 \\
Pneumonia & 08 & 4,8 \\
Empiema pleural & 04 & 2,4 \\
Atelectasia c/ pneumonia & 03 & 1.8 \\
Pneumonia c/ empiema & 02 & 1,2 \\
Atelectasia c/ empiema & 02 & 1,2 \\
\hline Total & 53 & 31,7 \\
\hline
\end{tabular}

As complicações decorrentes de falha no sistema de drenagem ocorreram em $35(20,9 \%)$ dos pacientes estudados. O hemotórax coagulado foi a complicação não infecciosa mais freqüente, estando presente em 21 $(12,6 \%)$ dos casos, seguido do pneumotórax residual com cinco $(2,9 \%)$ e derrame pleural com três $(1,7 \%)$ casos (Tabela 5).

Tabela 5

Número de casos conforme as complicações não infecciosas relacionadas com o procedimento cirúrgico

\begin{tabular}{lcc}
\hline Complicações Não Infecciosas & No de Casos & $\%$ \\
\hline Hemotórax residual & 21 & 12,5 \\
Pneumotórax residual & 05 & 3,0 \\
Derrame pleural & 03 & 1,8 \\
Obstrução do dreno & 03 & 1,8 \\
Saída do dreno & 02 & 1,2 \\
Fuga de ar & 01 & 0,6 \\
\hline Total & 35 & 20,9 \\
\hline
\end{tabular}

Após a análise bivariada, as seguintes variáveis correlacionadas com a ocorrência das complicações pleuropulmonares (pneumonia e empiema pleural) tiveram valor prognóstico: tipo de trauma (aberto ou fechado), tempo de drenagem pleural e presença de hemotórax coagulado (Tabelas 6 e 7). 
Tabela 6

Valores de p na análise bivariada

\begin{tabular}{l|c|c}
\hline Complicação & Pneumonia & Empiema \\
\hline $\begin{array}{l}\text { Fator de risco } \\
\text { Tipo de trauma }\end{array}$ & 0,05 & NS \\
Tempo de drenagem & 0,0074 & 0,0001 \\
\hline
\end{tabular}

Tabela 7

Número de casos conforme a ocorrência de complicações com e sem hemotórax coagulado

\begin{tabular}{lcc|c}
\hline Complicação & CPP/Sim & CPP/Não & Total \\
\hline $\begin{array}{l}\text { Fator de risco } \\
\text { Com hemotórax coagulado }\end{array}$ & 11 & 10 & 21 \\
Sem hemotórax coagulado & 42 & 104 & 146 \\
\hline Total & 53 & 114 & 167 \\
\hline
\end{tabular}

$\mathrm{CPP}=$ Complicação pleuropulmonar $\mathrm{p}=0,03$

Submeteram-se à análise multivariada apenas as variáveis que apresentaram, ao emprego da regressão logística univariada, correlação estatisticamente significativa com as variáveis atelectasia, pneumonia e empiema pleural. Em razão do pequeno número destas, quando tomadas isoladamente em cada um dos seus tipos, a análise multivariada foi efetivada considerando o total das mesmas, que foram agrupadas como variável dependente "complicações pleuropulmonares", proporcionando, assim, um melhor "balanceamento" dos dados, com resultados mais fidedignos ${ }^{10}$. Com este procedimento foi obtido o seguinte modelo (Tabela 8$)$.

\section{DISCUSSÃO}

O trauma, de modo geral, lesa indistintamente várias estruturas corporais e os traumatismos de tórax, em particular, exigem diagnóstico precoce e tratamento imediato em razão das alterações na dinâmica respiratória e circu- latória, tornando imprescindível uma avaliação rápida das condições clínicas do paciente, com ênfase na oxigenação adequada, na reexpansão pulmonar através da drenagem pleural, no tratamento do choque pela reposição volêmica e no controle da hemorragia, assim como no tratamento das lesões associadas ${ }^{17}$.

A probabilidade da ocorrência de infecção do espaço pleural, após este tipo de trauma, é dependente de uma complexa interação entre vários fatores de risco, incluindo o grau de contaminação bacteriana, a resistência do hospedeiro, a presença de corpo estranho na cavidade, o mecanismo da lesão e a presença de choque hemorrágico à admissão, entre outros 18, 19, 20 .

$\mathrm{Na}$ literatura, a presença de hemotórax residual (coagulado), com incompleta expansão pulmonar, tem sido referida como o principal fator de complicação infecciosa, incidindo em $5 \%$ a $30 \%$ dos casos. Sangue na cavidade torna-se um excelente meio de cultura para bactérias que chegam ao espaço pleural através da ferida torácica ou lesão pulmonar. O diagnóstico acurado e o tratamento precoce reduzem a morbidade e a mortalidade desta complicação ${ }^{8,21,22}$. Embora o hemotórax represente o primeiro evento após uma lesão penetrante do tórax, ele não consiste exclusivamente no único fator predisponente de infecção do espaço pleural, isto em razão da capacidade de reabsorção espontânea de pequenos volumes de sangue na ausência de infecção.

$\mathrm{Na}$ presente casuística, esta foi a complicação não infecciosa mais freqüente, ocorrendo em 21 (12,6\%) pacientes, nos quais o dreno não foi efetivo para evacuação adequada do sangue coletado. O radiograma de tórax evidenciou presença de áreas de parênquima pulmonar entremeadas com áreas de opacificação na base, imagem que equivale ao coágulo. No entanto, estas alterações radiográficas podem ser confundidas com outros processos pleurais, como: condensações pneumônicas, empiema, atelectasia e hérnia diafragmática ${ }^{21}$. Do total de 21 pacientes com hemotórax coagulado, 08 (4,7\%) evoluíram com algum tipo de complicação pleuropulmonar de caráter infeccioso, excluindo-se as atelectasias $(\mathrm{p}=0,03)$. No grupo de nove pacientes com coágulo retido nos quais não foi administrada a cefalotina, três evoluíram com empiema pleural, um com pneumonia, dois com atelectasia+empiema e um com pneumonia+empiema. Os outros dois pacientes apresentaram apenas atelectasia sem evolução infecciosa. No

Tabela 8

Resultado final da regressão logística para complicações pleuropulmonares

\begin{tabular}{l|c|c|c|c|c|c}
\hline Variável & Parâmetro & $D P$ & Qui-Quadrado & P-Valor & Odds Ratio & IC 95\% \\
\hline Intercepto & 0.6974 & 0.9299 & 0.5624 & 0.4533 & - & - \\
Tempo de internação & 0.3044 & 0.0728 & 17.4963 & 0.0001 & 1.356 & $1.176-1.564$ \\
Tipo de trauma & -2.8973 & 0.8332 & 12.0908 & 0.0005 & 0.055 & $0.011-0.282$ \\
Volume de sangue > 500ml & -0.9639 & 0.3994 & 5.8245 & 0.0158 & 0.381 & $0.174-0.834$ \\
\hline
\end{tabular}


grupo de 12 pacientes que receberam o antibiótico, apenas um, portador de hemotórax coagulado, evoluiu com pneumonia $(\mathrm{p}=0,002)$. Isto mostra a importância deste tipo de complicação como fator de risco de infecção pleuropulmonar.

Lesões mais graves do tórax, também contribuem para ocorrência de complicações pleuropulmonares. Estas, resultam freqüentemente em contusão pulmonar com hematoma e laceração do parênquima. Fraturas de arcos costais, com instabilidade da parede, cursam com quadro doloroso que, limitando os movimentos respiratórios, dificultam os mecanismos de "limpeza" da árvore traqueobrônquica e favorece $o$ aparecimento de atelectasia com evolução pneumônica 20,23

As atelectasias, são referidas na literatura, como complicações pleuropulmonares de maior incidência nos pacientes politraumatizados, em especial quando há comprometimento da mecânica ventilatória. De manifestação frustra, esta complicação, na maioria das vezes, cursa com pouco ou nenhum sintoma, sendo pouco detectada pelo exame clínico de rotina. $\mathrm{O}$ alto índice de suspeição, conjuntamente com o radiograma do tórax, evidenciam sua presença, aumentando, acentuadamente, sua incidência ${ }^{22}$, ${ }^{23}$. Esta complicação esteve presente em 39 (23,3\%) dos pacientes estudados, não interferindo na média e desvio-

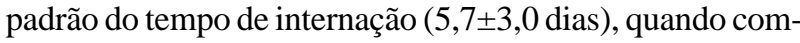
parada com a dos que evoluíram sem esta complicação (5,6 63,1 dias). Sua evolução para complicações mais graves não mostrou significância estatística.

O prolongamento do tempo de drenagem parece ser outro fator importante no desenvolvimento de complicações. Múltiplos estudos mostraram que a permanência do dreno pleural por um período acima de 72 horas, aumenta a incidência de infecção do espaço pleural. Neste sentido, o dreno deverá ser removido tão logo seja possível, desde que tenha cumprido sua função. Neste trabalho, a média de permanência do dreno, na cavidade pleural, foi de $5,1 \pm 2,8$ dias, compatível com os dados da literatura consultada $12,20,24$.

A técnica cirúrgica empregada, quando da inserção do dreno, é fundamental para a profilaxia da infecção, devendo-se instituir todos os cuidados de assepsia e anti-sepsia, evitando-se, com isto, o carreamento de bactérias para o espaço pleural ${ }^{20,25}$. Assim, a contaminação, pode ocorrer não somente pelo próprio mecanismo do trauma, mas, também, pela inobservância dos princípios técnicos no momento da colocação do dreno.

A presença de corpo estranho no interior da cavidade torácica é outro componente a ser observado, que aumenta a possibilidade de infecção ${ }^{22}$. Portanto, cuidado especial deve ser dispensado para identificação e remoção dos mesmos, quando introduzidos no espaço pleural, em conseqüência ao trauma, sob o risco de infecção, mesmo após drenagem e antibioticoterapia adequadas ${ }^{26}$. Neste trabalho, a opção pela drenagem pleural, conforme a técnica preconizada pelo ATLS, considerou ser esta a mais apropriada para tratar pacientes com trauma torácico, sendo, atualmente, a mais empregada, em razão da menor incidência de complicações associadas.
O mecanismo do trauma deve ser olhado com especial atenção. A presença de tecido necrótico na parede ou no interior da cavidade torácica aumenta a incidência de empiema. Por esta razão, os ferimentos causados por projéteis de armas de fogo, que levam à maior destruição tecidual, são mais propícios à infecção, quando comparados aos ferimentos por arma branca ${ }^{20}$. Na presente pesquisa, observou-se que os pacientes que sofreram traumatismo fechado evoluíram com maior incidência de complicações pleuropulmonares quando relacionados a outros agentes vulnerantes.

A idade é também um fator de risco a ser considerado. Pacientes idosos, mais amiúde, apresentam diminuídas suas reservas fisiológicas cardiovascular e respiratória, freqüentemente com deonças crônicas associadas, estando, portanto, mais propensos a reter secreções pulmonares com desenvolvimento de atelectasias e pneumonias subseqüentes. Estes pacientes possuem maior grau de imunodepressão, com resposta deficiente ao trauma, quando comparados com a população mais jovem ${ }^{12,} 19$, 27. Ainda que as vítimas de lesões torácicas, em sua maioria, pertençam à faixa etária mais jovem, portanto com menor incidência de afecções associadas, o trauma em si induz a graus diversos de imunodepressão, colocando estes pacientes, igualmente, no grupo de risco para o desenvolvimento de complicações infecciosas ${ }^{23}$. Na presente casuística, $120(71,9 \%)$ dos pacientes avaliados pertenciam à faixa etária mais jovem da população, com idade compreendida entre 13 e 29 anos, portanto compatível com os dados relatados na literatura ${ }^{3,7,9,17}$.

Outros fatores associados ao trauma, do mesmo modo, podem contribuir para depressão do sistema imunológico. Procedimentos cirúrgicos, técnicas invasivas de diagnóstico ou terapêuticas, uso prévio de medicamentos (antibióticos, corticóides) ou drogas, como álcool e fumo, devem ser pesquisados ${ }^{20,23}$.

O risco subseqüente de infecção, também aumenta na presença de lesões associadas, com hemorragia e choque de duração prolongada à admissão e deterioração do nível de consciência por trauma cranioencefálico, ficando a prevenção da infecção do espaço pleural melhor determinada pela evacuação completa e retirada precoce do dreno torácico ${ }^{12,19,28}$. Ao correlacionarem-se os valores do RTS, ISS e do TRISS, no presente trabalho, com as complicações pós-operatórias, não se constatou suas aplicabilidades como índices de prognóstico, exceto que, no caso do TRISS, os valores encontrados refletiram a taxa de sobrevivência encontrada. As causas desta falta de aplicabilidade decorreram da seleção dos pacientes, com lesões restritas à região do tórax, produzindo valores com estreita margem de variação, entre nove e 16 pontos para o ISS e 97\% a 99\% para o TRISS.

$\mathrm{Na}$ análise bivariada de correlação das complicações pleuropulmonares com as demais variáveis, representadas pelos fatores de risco, pôde-se identificar duas delas com valor prognóstico estatisticamente significante: tipo de trauma e tempo de drenagem pleural. Entretanto, com este modelo de análise, nos é difícil concluir pela verdadeira importância de cada uma destas variáveis, quando toma- 
das isoladamente, na determinação do prognóstico; ou se as mesmas estão associadas entre si. A análise estatística de regressão logística multivariada foi utilizada para identificar a associação entre estas variáveis. Este modelo fornece a significância estatística juntamente com o valor prognóstico de cada variável, ajustadamente, considerando concomitantemente a contribuição das demais variáveis. Com este procedimento, quatro variáveis mostraram-se como fator preditivo de complicação pleuropulmonar: tempo de drenagem $(\mathrm{p}=0,0002)$; tempo de internação $(\mathrm{p}=$ $0,0001)$; tipo de trauma $(\mathrm{p}=0,0012)$ e mecanismo da lesão $(\mathrm{p}=0,0020)$.

O procedimento Stepwise foi utilizado para selecionar as variáveis que melhor explicavam a variável resposta complicação pleuropulmonar. Após esta análise, o modelo obtido pela regressão logística multivariada mostrou que as variáveis tempo de internação, tipo de trauma e volume de sangue drenado durante a cirurgia, maior do que $500 \mathrm{ml}$, foram as que mais influenciaram no aparecimento das complicações pleuropulmonares.

A odds ratio (razão de chance) utilizada para mensurar a associação entre os fatores de risco e a ocorrência de complicações pleuropulmonares confirmou ser o trauma fechado o fator mais significativo. Esta condição decorre da contusão pulmonar resultante de forças compressivas que atuam sobre o pulmão, produzindo rupturas capilares com formação de edema e extravasamento de líquido intravascular, prejudicando a aeração das áreas atingidas com formação de zonas de atelectasia. O tórax instável, associado ao trauma fechado com fratura de arcos costais, é outro fator que contribui para o aparecimento destas complicações em razão da limitação respiratória provocada pela dor. Neste caso, se a contusão e a dor são efetivamente controladas e a intubação orotraqueal é evitada, o risco de complicações pode ser minimizado ${ }^{23,29}$.

Analisando o modelo observado, podemos inferir que os pacientes com trauma fechado têm 18,1 vezes mais chances de evoluir com algum tipo de complicação pleuropulmonar do que aqueles portadores de trauma aberto; se o volume de sangue drenado, durante o procedimento da toracostomia, for superior a $500 \mathrm{ml}$, a chance aumenta em 2,6 vezes e, para estes pacientes, cada dia a mais no tempo de internação, aumenta em 1,3 vezes suas chances de evoluir com estas complicações.

O hemotórax coagulado foi a complicação relativa à falha no sistema de drenagem mais envolvida com a evolução para um quadro infeccioso.

\begin{abstract}
The objective of this study was to analyse the risk factors for the development of thoracic infections after tube thoracostomy. Although technically simple, this procedure, 1 to 25 percent of the patients develop some type of intra or post-operative complications. A total of 167 patients, submitted to emergency tube thoracostomy, were admitted and stratified into two groups selected by randomic sampling to a cohorts accompanying study. One hundred and four patients, without antibiotic therapy, were considered as been the control group; and, 63 patients using cefalotin in post-operative as the experimental group. The mean age of the patients in the control group was

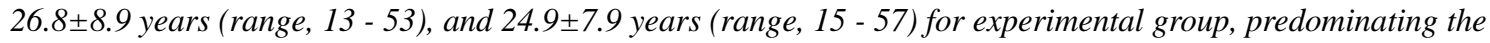
male sex (95.2\%) in both studied groups. The penetrating chest trauma was present in $92.8 \%$ of the patients, with a higher incidence of stab wounds $(58.7 \%)$ in contrast to gunshot wounds (24.6\%). Thoracic complications were present in 35 patients (33.7\%) of the control group, whereas, in the experimental group, only 18 patients (28.6\%) developed this kind of complication. In the statistic significance analysis, the bivariate model indicated that the variable trauma type and the duration of pleural space drainage were the most relevant ones as predictive factors for infections complications. In the multivariate logistic regression, the variables blunt chest trauma, length of hospital stay and drainage blood volume higher than $500 \mathrm{ml}$, when associated, influenciated positively on the occurrence of these complications.
\end{abstract}

Keywords: Trauma; Thoracic trauma; Pleural drainage; Risk factors; Logistic regression.

\title{
REFERÊNCIAS
}

1. Schwartz SI : Princípios de Cirurgia. 5. ed. Rio de Janeiro, Guanabara Koogan, 1993. 2v. 1957p.

2. Breasted JH : The Edwin Smith Papyrus, Chicago, Ill., 1930, The University of Chicago Press, vol. I.

3. LoCicero III J, Mattox KL : Epidemiology of Chest Trauma. Surg Clin North Am 1989; 69:15 - 9.

4. Calhoon JH, Trinkle JK : Pathophysiology of Chest Trauma. Chest Surg Clin N Am 1997; 7:199 - 211.
5. Block FJ, Kirton OC, Windsor J, et al. : Guided Percutaneous Drainage for Posttraumatic Empyema. Thoracic Surg 1995; 117:282 - 7.

6. Sabiston Jr, David C : Tratado de Cirurgia: As Bases Biológicas da Prática Cirúrgica Moderna. 14. ed. Rio de Janeiro, Guanabara Koogan, 1993. 2v. 2133 p.

7. Silas MG, Belluzzo BR, Miguel EG : Traumatismo Torácico: análise de 231 casos. Arq Med ABC 1990; 13:19 - 21. 
8. Symbas PN : Chest Drainage Tubes. Surg Clin North Am $1989 ; 69: 41-46$.

9. American College of Surgeons. Manual de Suporte Avançado de Vida no Trauma - ATLS. 5. ed., rev. s. 1: Ministério da Saúde, 1993. 414 p.

10. Jekel JF, Elmore JG, Katz DL : Epidemiologia, Bioestatística e Medicina Preventiva. Porto Alegre, Artes Médicas Sul, 1999. 328 p.

11. Grover FL, Richardson JD, Fewel JG, et al. : Prophylatic antibiotics in the treatment of penetrating chest wounds. J Thorac Cardiovasc Surg 1977; 74:528-36.

12. LoCurto JJ, Tischler CD, Swan KG, et al. - Tube thoracostomy and trauma - antibiotics or not? J Trauma 1986 ; 26:1067-72.

13. Garner JS, Jarvis WR, Emori TG, et al. : CDC definitions for nosocomial infections, 1988. Am J Infect Control 1988; 16:128-40.

14. Dripps RD, Deming MVN : Postoperative atelectasis and pneumonia. Ann Surg 1946, 124:94-110.

15. Felson B : Radiologia toracica. 2 ed. Corrigida. Barcelona, Editorial Cientifica - Medica, 1985. 507 p.

16. Bernini C O : Ferimentos toracoabdominais: estudo de fatores determinantes de complicações pleuropulmonares. Tese de Doutorado, 1992. Faculdade de Medicina - USP.

17. Fragomeni LS, Pertuzzatti ML, Falleiro R, et al. : Trauma Torácico: manejo clínico-cirúrgico. An Med Cir 1994; $6: 38-42$

18. Borja AR, Ransdell HR : Treatment of Penetrating Gunshot Wounds of the Chest. Am J Surg 1971; 122:81 - 5 .

19. Walker WE, Kapelanski DP, Weiland AP, et al. : Patterns of Infection and Mortality in Thoracic Trauma. Ann Surg $1985 ; 201: 752-757$.

20. Eddy AC, Luna GK, Copass M : Empyema Thoracic in Patients Undergoing Emergent Tube Thoracostomy for Thoracic Trauma. Am J. Surg 1989; 157:494 - 97.

21. Tarantino AB : Doenças Pulmonares. 2 ed. Rio de Janeiro, Guanabara Koogan, 1982. 907 p.

22. Richardson JD, Miller FB, Carrillo EH : Complex Thoracic Injuries. Surg Clin North Am 1996; 76:725 - 46.
23. McRitchie DI, Matthews JG, Fink MP : Pneumonia in Patients With Multiple Trauma. Clin Chest Med 1995; $1: 135-46$.

24. Stone HH, Symbas PN, Hooper CA : Cefamandole for Profilaxis Against Infection in Closed Tube Thoracostomy. J Trauma 1981; 21:975 - 977.

25. Millikan JS, Moore EE, Steiner E, et al. : - Complications of Tube Thoracostomy for Acute Trauma. Am J Surg $1980 ; 140: 738-41$.

26. Mandal AK, Thadepalli H, Mandal AK, et al. : Posttraumatic Empiema Thoracic: A 24 - Year Experience at a Major Trauma Center. J Trauma 1997; 5:764 - 71.

27. Sriussadaporn S, Poomsuwan P : Post-Traumatic Empiema Thoracis in Blunt Chest Trauma. J Med Assoc Thai 1995; 78:393 - 398.

28. Etoch SW, Bar-Natan MF, Miller FB, et al. : Tube Thoracostomy: factors related to complications. Arch Surg $1995 ; 130: 521-26$.

29. Trinkle JK, Richardson JD, Franz JL : Management of Flail Chest Without Mechanical Ventilation. Ann Thorac Surg 1975; 19:355 - 60 .

Endereço para Correspondência

Dr. Mauro José Fontelles

Rua Major Solon, 615 / 62 - Cambuí

13024-907 - Campinas-SP 